\title{
A Repetitive-PI Current Controller for Boost Single Phase PFC Converters
}

\author{
Turki Kahawish Hassan \\ Electrical Engineering Department, University of AL-Mustansiriya, Baghdad, Iraq \\ E-mail: turkihassan@yahoo.com \\ Received November 27, 2010; revised January 4, 2011; accepted January 26, 2011
}

\begin{abstract}
In this paper, the Author presents the theory and application of repetitive proportional integral current controller for boost single phase ac-dc converter with power factor correction (PFC). A repetitive controller which is inserted in series with the proportional integral (PI) controller shows very low crossover distortion of input current, low total harmonic distortion and very low tracking error when is compared with the conventional proportional integral controller. Full analysis of proposed controller is given and Matlab/Simulink is used for simulation. The simulation results show the validity of the proposed control method.
\end{abstract}

Keywords: Boost Converter, Unity Power Factor Correction, Repetitive Control

\section{Introduction}

The developments in power factor correction technology in the past two decades have enabled the design and implementation of single phase ac-dc boost converters with close to unity power factor and much less input current distortion that generated by simple diode rectification circuits.

Typical PFC schemes use two cascaded loops: the first loop aims to regulate the boost $\mathrm{dc}$ output voltage and provide the amplitude for the reference to the second controller, which controls sinusoidal current provided from grid. This current always contains some residual distortion, especially in the vicinity of zero crossing of the input voltage. The reasons of this zero crossing distortion (also referred to as cross distortion) are discussed by authors in $[1,2]$ but no any effective method is suggested to reduce or eliminate this phenomenon. Feed forward current control methods for boost single phase PFC converters are suggested for robust control against parameters variation and to reduce the total harmonic distortion $[3,4]$.These methods need additional circuits and the cost is increased .

Receptive control theory [5-7] originating from internal model principle provides a solution for eliminating periodic errors which occur in a dynamic system. A repetitive controller can be viewed as a periodic waveform generator augmented within the control loop of a control system, which is closed loop regulated by a feed back controller so that the periodic errors can be eliminated. A number of repetitive control schemes have been developed and applied to eliminate periodic distortions in PWM inverters $[8,9]$.

In this paper, a repetitive controller is proposed and designed to eliminate the zero crossing distortion of input current of the converter and to obtain good tracking performance. The design of the repetitive controller is performed by analyzing the frequency domain, and $\mathrm{Ny}$ quist plot play a central role throughout the design phase. The feasibility of the proposed design technique is shown by simulation. The simulation results of the proposed system show a highly improvement compared to the conventional PI controlled system.

The reminder of this paper is organized as follows. Section 2 gives operation principle of boost single phase PFC converter and formulates the problem. In Section 3, the principle of repetitive control is reviewed and a repetitive controller for PFC boost converter is proposed. In section 4 , the repetitive controller is designed and several simulation results for proposed and conventional systems are presented. Section 5 concludes the paper.

\section{Operation Principe and Problem Formulation}

Power circuit configuration of a boost single-phase power factor correction (PFC) converter and its control scheme are shown in Figure 1(a) and (b) respectively. 


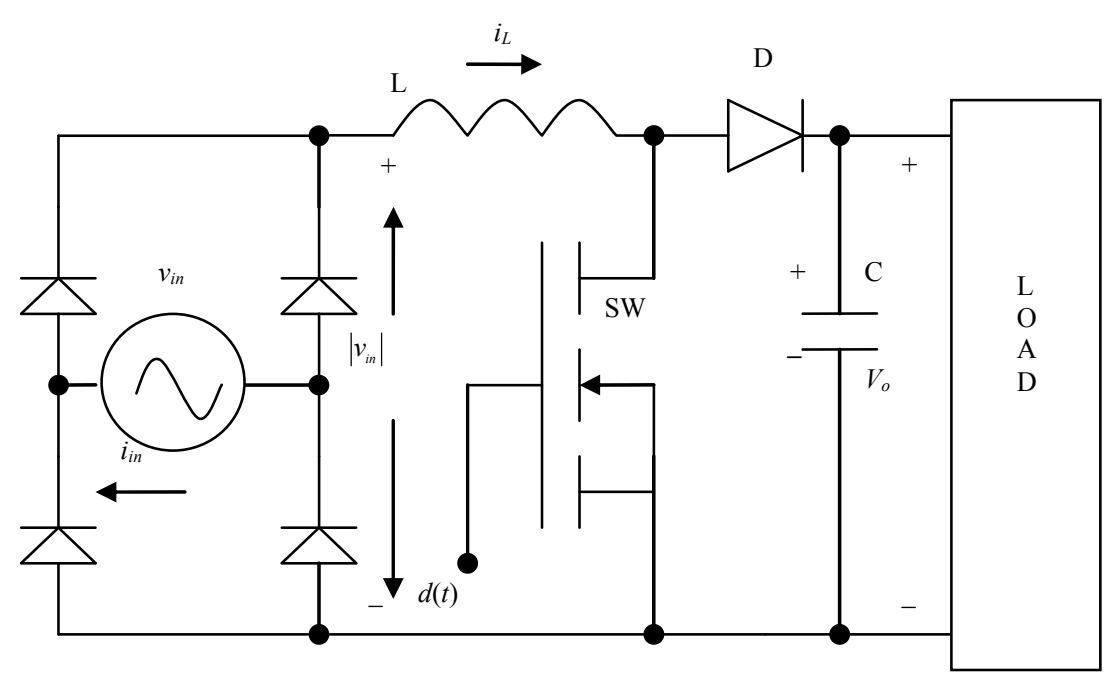

(a)

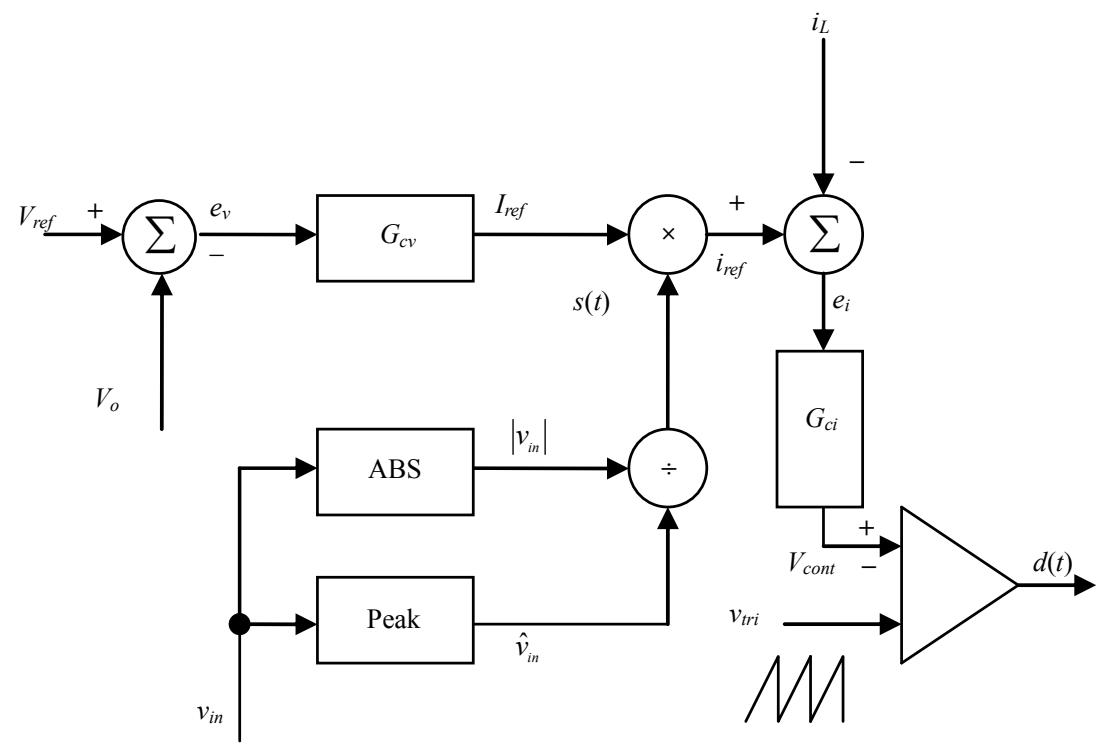

(b)

Figure 1. Boost single phase PFC converter (a) power circuit and (b) control block diagram.

The circuit consists of a full-bridge diode rectifier and a boost $\mathrm{dc} / \mathrm{dc}$ converter. The dc output voltage of the boost converter $V_{o}$ is compared with the reference $V_{\text {ref }}$ and the error $e_{v}$ is applied to the voltage controller $G_{c v}(s)$ which is a PI controller. A current command $i_{\text {ref }}$ is yielded by multiplying the output of voltage controller $I_{r e f}$ with a rectified unity sine wave $(s(t))$ which is:

$$
s(t)=\frac{\left|v_{\text {in }}\right|}{\hat{v}_{\text {in }}}=|\sin \omega t|
$$

where $v_{i n}=\hat{v}_{i n} \sin \omega t, \hat{v}_{i n}$ is the amplitude of input voltage.

The current command is compared with the inductor current $i_{L}$ and the error $e_{i}$ is applied to the PI current controller $G_{c i}(s)$ to obtain $V_{\text {cont }}$. A pulse width modulation control signal $\mathrm{d}$ which is obtained by comparing $V_{\text {cont }}$ with the triangular wave $V_{t r i}$ is applied to gate of power MOSFET transistor to achieve the sinusoidal input current $i_{\text {in }}$.

In order to make the following control system analysis and design, some reasonable assumptions are made:

1) the losses of converter components are neglected;

2) the dc output voltage is well regulated such that $V_{o}$ equals to reference value $V_{\text {ref. }}$.

Accordingly one can derive the following equation using the state-space averaging method.

$$
L \frac{\mathrm{d} i_{L}}{\mathrm{~d} t}=\hat{v}_{i n}|\sin \omega t|-(1-d(t)) V_{r e f}
$$

where $L$ is the boosting inductance and $d(t)$ is the duty 
ratio function of the PWM modulator which can be expressed as

$$
d(t)=\frac{V_{\text {cont }}}{\hat{V}_{\text {tri }}}
$$

where $\hat{V}_{t r i}$ is the amplitude of the triangular wave. From (2) and (3), one can find

$$
\frac{\mathrm{d} i_{L}}{\mathrm{~d} t}=\frac{\hat{v}_{i n}|\sin \omega t|}{L}-\frac{V_{r e f}}{L}+\frac{V_{r e f}}{L \hat{V}_{t r i}} V_{c o n t}
$$

As shown in Figure 1(b), $V_{\text {cont }}$ can be written in laplace form as

$$
V_{\text {cont }}(s)=\left(i_{\text {ref }}(s)-i_{L}(s)\right) G_{c i}(s)
$$

The inductor current $i_{L}$ can be derived from (4) and (5) to be

$$
i_{L}(s)=\frac{\left|v_{i n}\right|(s)}{s L(1+G(s))}-\frac{V_{r e f}}{s^{2} L(1+G(s))}+\frac{G(s)}{1+G(s)} i_{r e f}(s)
$$

where

$$
G(s)=\frac{V_{r e f}}{s L \hat{V}_{t r i}} G_{c i}(s)
$$

and

$$
G_{c i}(s)=K_{p}+\frac{K_{i}}{s}
$$

where $K_{p}$ and $K_{i}$ are proportional and integral gains respectively.

According to (6), the absolute value of input voltage can be viewed as a disturbance to the control loop. Also $V_{\text {ref }}$ can be considered as a constant disturbance. The objective of control is to make the inductor current tracks a sinusoidal reference current, in face of these disturbances. In the conventional control method, a proportional integral (PI) controller $G_{c i}(s)$ is used in design and implementation, and also the most commonly used one in practical applications. However, it fails to yield excellent control performances in both tracking and disturbance rejection issues. To solve these problems, a repetitive controller is proposed and applied to the current loop of boost converter. This controller is discussed in next section.

\section{Repetitive Control}

\subsection{Principle of Repetitive Control}

Any periodic signal with period Ts can be generated by the free time delay system. The block diagram of time delay system including unity positive feedback is shown in Figure 2(a). The resulting transfer function is [5]:

$$
G_{R}(s)=\frac{y(s)}{u(s)}=\frac{e^{-s T_{S}}}{1-e^{-s T_{s}}}
$$

where $y(s)$ is the output and $u(s)$ is the input. Due to this delay, the transfer function has infinitely many poles on the imaginary axis: $j k \omega_{s}$ (see Figure 2(b)). The poles can be found from $\left.e^{-s T_{s}}\right|_{s=j k \omega s}=1.0$ for every $k=0, \pm 1$, $\pm 2, \cdots$, where $\omega_{s}=2 \pi / T_{s}$.

A controller including the model in (9) is said to be a repetitive controller and a system with such controller is called a repetitive control system.

The basic concept of the repetitive controller originates from the internal model principle [7]. This principle state that the controlled output tracks a set of reference inputs without steady state error if the model which generates these references is included in the stable closed loop system. For example, no steady state error occurs for step reference commands in type-1 stable feedback system that has an integrator $(1 / s)$ in the loop, i.e., the generator of step function .However, stand-alone repetitive controller cannot yield good transient performance, therefore the repetitive controller is often used together with another controller such as P-I controller to give quick transient response.

\subsection{PFC Boost Converter with Repetitive Controller}

The block diagram of the proposed repetitive control system is shown in Figure 3. The repetitive controller $C_{R P}(s)$ is located in series with conventional PI controller $G_{c i}(s)$ of current loop of boost converter. The transfer function of the repetitive controller is:

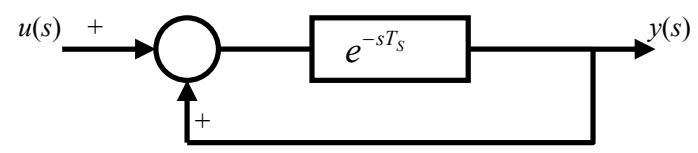

(a)

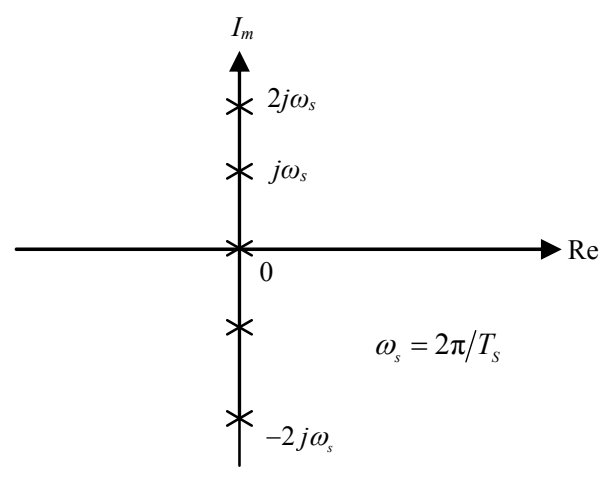

(b)

Figure 2. Generator of periodic signal. 


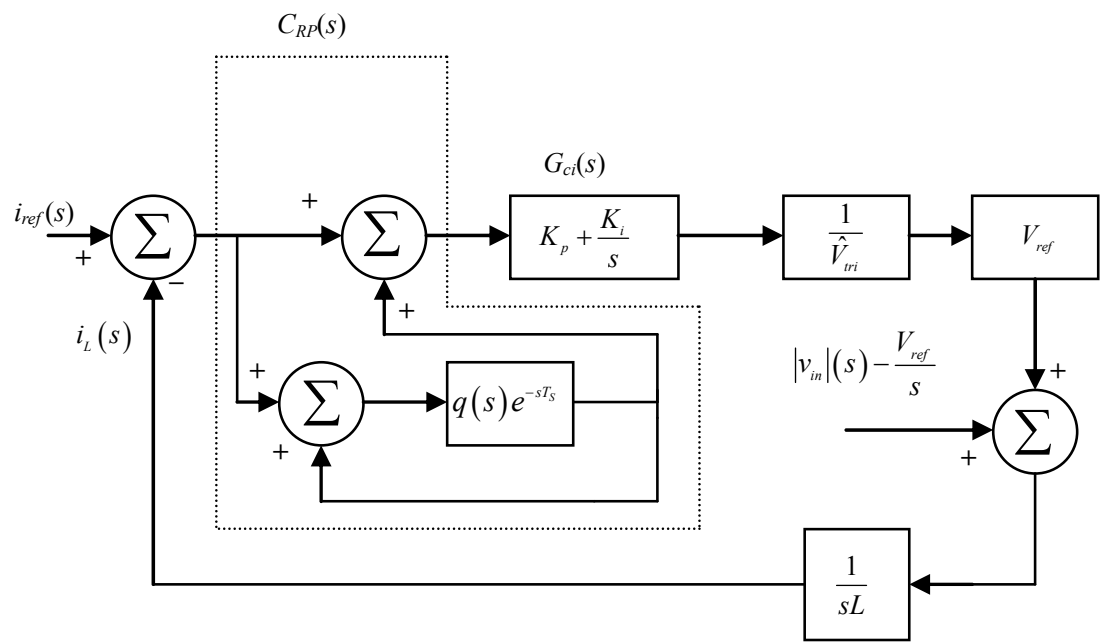

Figure 3. Block diagram of a repetitive control system.

$$
C_{R P}(s)=\frac{1}{1-q(s) e^{-s T_{S}}}
$$

Note that $C_{R P}(s)$ is equivalent to the modified repetitive controller with $a(s)=1$ as proposed by Hara et al. [5]. $q(s)$ is a low pass filter that should be appropriately chosen so that good tracking performance is obtained without resultant system instability. The choice of $q(s)$ is not straightforward and simulations are needed to choose the most appropriate filter [10].

For the proposed repetitive control system, (6) can be rewritten as:

$$
\begin{aligned}
i_{L}(s)= & \frac{\left|v_{i n}\right|(s)}{s L\left(1+G_{R P}(s)\right)}-\frac{V_{r e f}}{s^{2} L\left(1+G_{R P}(s)\right)} \\
& +\frac{G_{R P}(s)}{1+G_{R P}(s)} i_{r e f}(s)
\end{aligned}
$$

where

$$
G_{R P}(s)=\frac{V_{r e f}}{s L \hat{V}_{t r i}} G_{c i}(s) C_{R P}(s)
$$

Let Us discuss the effectiveness of the repetitive controller according to (11) and (12). The transfer function $C_{R P}(s)$ is large enough for $s=j k \omega_{s}, k=0,1,2, \cdots$ If the proportional integral controller $G_{c i}(s)$ of current loop of boost converter is chosen such that $\left|G_{R P}\left(j k \omega_{s}\right)\right|$ is sufficiently large for the selective range of harmonics which are multiples of frequency of reference current, then $1+G_{R P}\left(j k \omega_{s}\right) \approx G_{R P}\left(j k \omega_{s}\right)$. Since the denominator is large, the disturbances are rejected and the inductor current tracks the sinusoidal reference current with very small steady state error.

The stability condition that is obtained in [5] is applied to proposed repetitive control system to give the follow- ing proposition:

In the repetitive control system shown in Figure 3 if the closed loop system without repetitive controller, i.e., $G(s) /(1+G(s))$, is stable and $|q(j \omega)|<|1+G(j \omega)|$ $\forall \omega \geq 0$ where

$$
G(s)=\frac{V_{r e f}\left(K_{p} s+K_{i}\right)}{s^{2} \hat{V}_{t r i} L}
$$

then the system is exponentially stable.

The system without repetitive controller is stable since $G(s)$ has no unstable poles. The repetitive control system is therefore exponentially stable if the Nyquist plot of $G(s)$ [11] does not encircle the $(-1, j 0)$ point and lies outside of the circle of radius $|q(j \omega)|$ centered at the $(-1, j 0)$ point in the complex plane. The low pass filter $q(s)$ should be chosen so that the Nyquist plot of $G(s)$ remains outside the circle for all frequencies.

\section{Controller Design and Results}

\subsection{Controller Design}

The boost single phase ac-dc converter with power factor correction has outer voltage loop and inner current loop. Some nominal values and circuit parameters of the converter are shown in Table $\mathbf{1 .}$

Our investigation is focused on the inner current loop with repetitive controller that is shown in Figure 3. The parameters of P-I controller of current loop are chosen as $K_{p}=0.8$ and $K_{i}=300$. The repetitive controller is inserted in series with the PI controller.

Figure 4 shows the Nyquist plot of $G(s)$. The plot does not encircle the $(-1, j 0)$ point and for stable system the plot must be outside the circle of radius of $|q(j \omega)|$. To ensure the stability for all frequencies the value of 


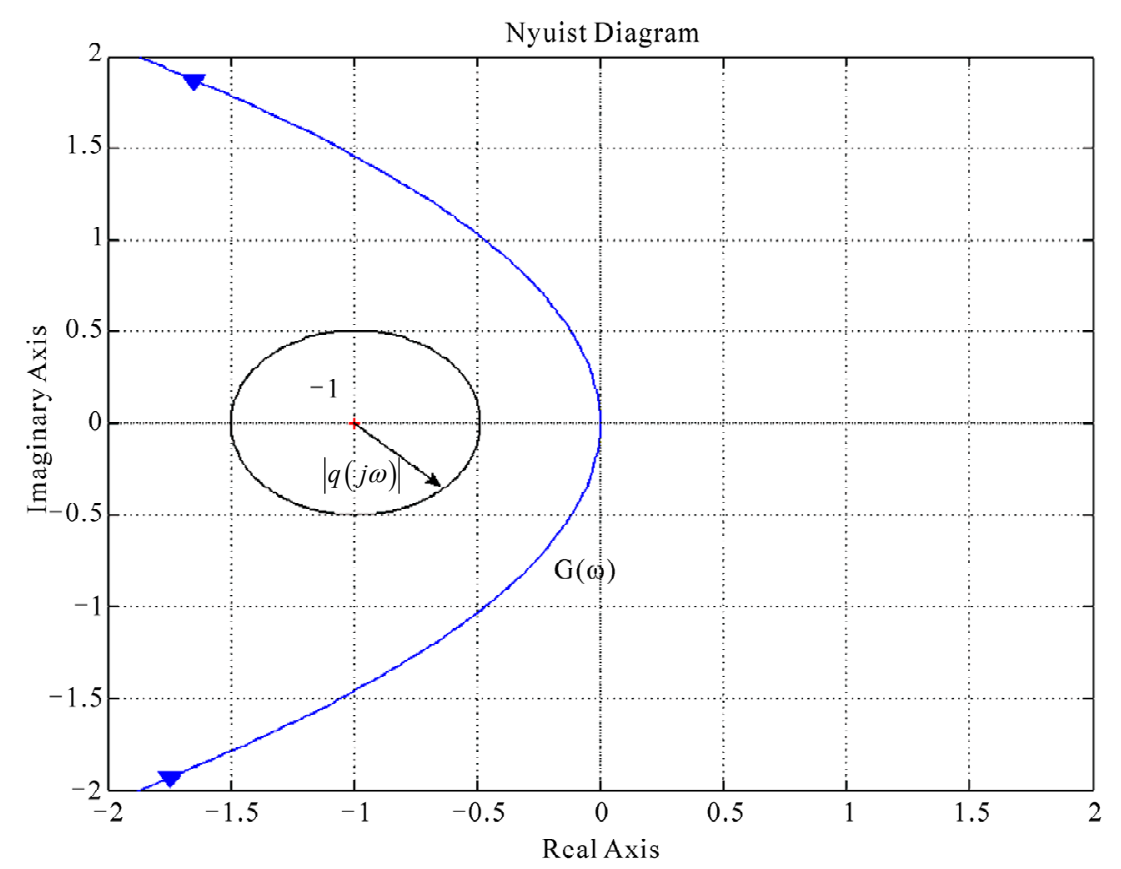

Figure 4. Stability circle and Nyquist plot of G(s).

Table 1. Simulated converter parameters.

\begin{tabular}{ll}
\hline Input line voltage (peak) & $\hat{v}_{\text {in }}=170 \mathrm{~V}$ \\
Input line frequency & $\mathrm{f}=50 \mathrm{~Hz}$ \\
Smoothing capacitance & $\mathrm{C}=1000 \mu \mathrm{F}$ \\
Smoothing inductance & $\mathrm{L}=1 \mathrm{mH}$ \\
Rated power & $500 \mathrm{~W}$ \\
Carrier frequency & $25 \mathrm{KHz}$ \\
Output voltage & $V_{o}=300 \mathrm{~V}$ \\
\hline
\end{tabular}

$|q(j \omega)|$ must be less than unity to prevent any contact between the plot of $G(s)$ and the circle (see the stability condition in the previous section). The gain of low pass filter $q(s)$ is chosen as 0.98 to give stable system and good tracking performance.

No systematic method to obtain the cutoff frequency of $q(s)$, therefore simulation is required to find the best value from the point of view of good tracking and disturbances rejection. Accordingly, the cutoff frequency is chosen as $1000 \mathrm{HZ}$. It seems that $1000 \mathrm{HZ}$ is reasonable choice since most periodic disturbances are expected to lie within this band. Let the filter $q(s)$ be

$$
q(s)=\frac{0.98}{1+s / 2000 \pi}
$$

In our applications, we require a delay of $T_{s}=10 \mathrm{~ms}$ for compensation of harmonics of $f_{s}=100 \mathrm{~Hz}$. Finally, we get a repetitive controller $C_{R P}(s)$, which has transfer function

$$
C_{R P}(s)=\frac{1}{1-\frac{0.98}{1+s / 2000 \pi} e^{-s / 100}}
$$

The effects that the designed repetitive controller $C_{R P}(s)$ has on the PFC boost converter system can be investigated by the bode plots of loop gains $G(s)$ and $G_{R P}(s)$ (without and with repetitive controller respectively) shown in Figure 5.

The major characteristics of the repetitive controller, such as effective rejection of periodic disturbances and reduction of stability margin, are clearly seen from the Bode plots. Note that the added repetitive controller increases the loop gain at particular frequencies, integral multiples of $100 \mathrm{~Hz}$, maintaining a relatively unchanged gain at other frequencies. Let us considerer the sensitiveity function $\frac{1}{1+G_{R P}(s)}$ which is the transfer function from disturbance input to tracking error, which is of primary importance in judging the performance of feedback control systems. Gain increase at particular frequencies leads to decrease in sensitivity at these frequencies. This implies that zero crossing distortion in inductor current which is considered as the periodic disturbances appearing at multiple of $100 \mathrm{~Hz}$ are more strongly attenuated by the repetitive controller than non periodic disturbances.

\subsection{Results}

Simulation is performed by Matlab/Simulink to verify 

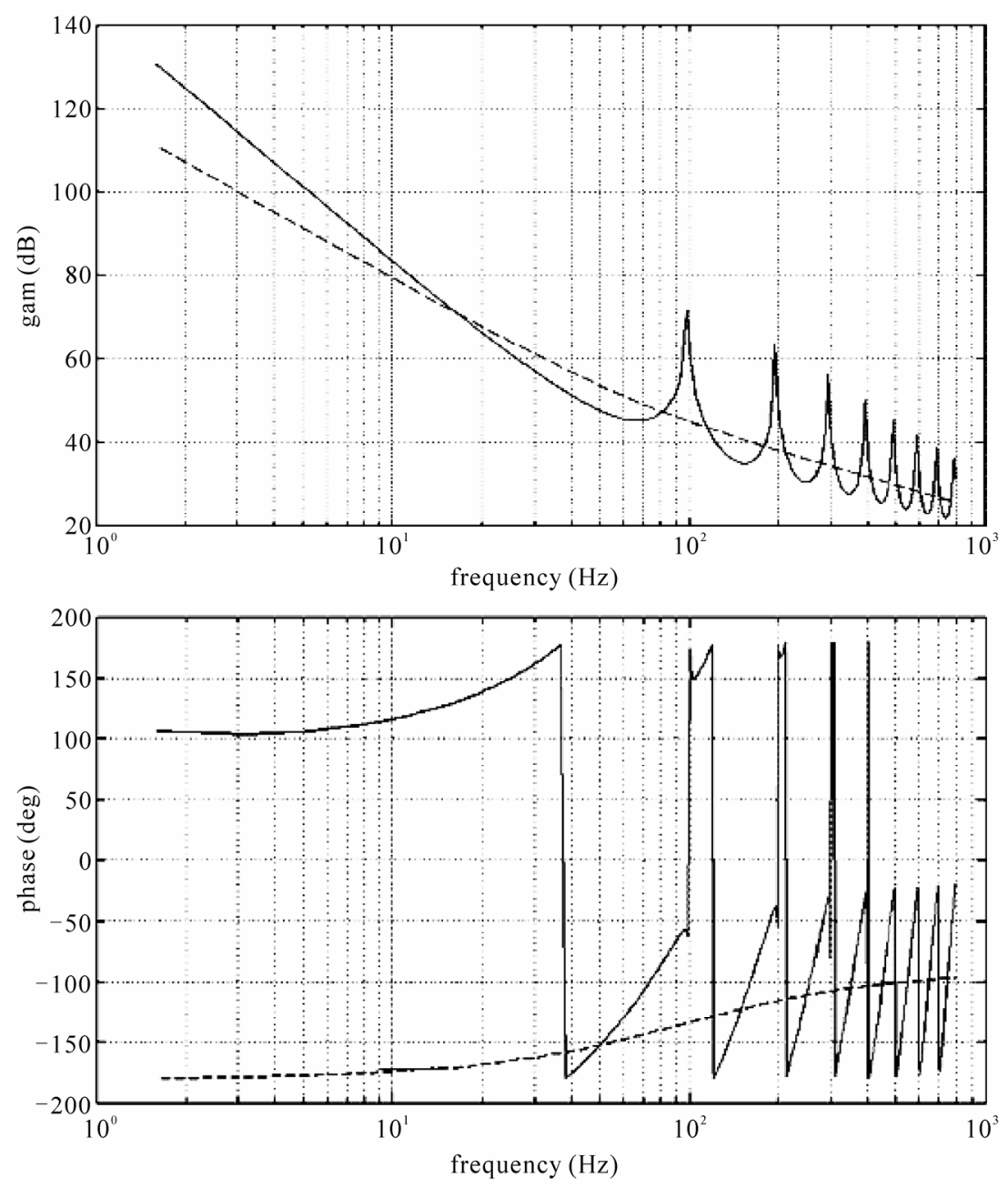

Figure 5. Bode plots of $G(s)$ with (dash) and $G_{R P}(s)$ with solid.

the proposed PFC boost converter with repetitive controller. The converter parameters in Table $\mathbf{1}$ and the designed repetitive controller in the previous section are used in simulation. For all simulation results, the output voltage of the converter is constant and equals to $300 \mathrm{~V}$.

Figure 6(a) and (b) show the input current $i_{\text {in }}$ and the input voltage $v_{i n}$ waveforms with and without repetitive controller respectively for $100 \mathrm{~W}$ output power and load resistance $\left(R_{l}\right)$ of $900 \Omega$. The input current is in phase with input voltage and has an amplitude of $1.18 \mathrm{~A}$ with no zero switching distortion when repetitive controller is used.

Figure 7(a) shows the reference current $i_{\text {ref }}$ and the inductor current $i_{L}$ waveforms with $100 \mathrm{~W}\left(R_{\mathrm{l}}=900 \Omega\right)$ when the repetitive controller is applied. Comparison with waveforms in Figure 7(b) when only PI-controller is used indicates that current tracking performance is significantly improved by the proposed repetitive controller (reference and inductor current waveforms are coincident.
Figure 8(a) shows the input voltage and input current for $R_{l}=450 \Omega$ (output power equals $200 \mathrm{~W}$ ) with repetitive controller. It is noticed that the zero crossing distortion in input current is canceled compared with Figure 8(b) when only P-I controller is used.

Zero crossing distortion in input current shown in Figure 6(b) is more than the distortion shown in Figure 8(b) because of increment in load current with load resistance of $450 \Omega$.More leading phase of input current when load resistance is increased (less load current) which causes more zero crossing distortion [1]. This leading phase is not appeared when the proposed repetitive controller is used.

Figure 9(a) and (b) show the reference and inductor current waveforms for load resistance of $450 \Omega$ with and without repetitive controller respectively. It is clear that a good tracking is achieved with repetitive controller (very small steady state error between reference and inductor currents).

Figure 10(a) and (b) show the input voltage and input 


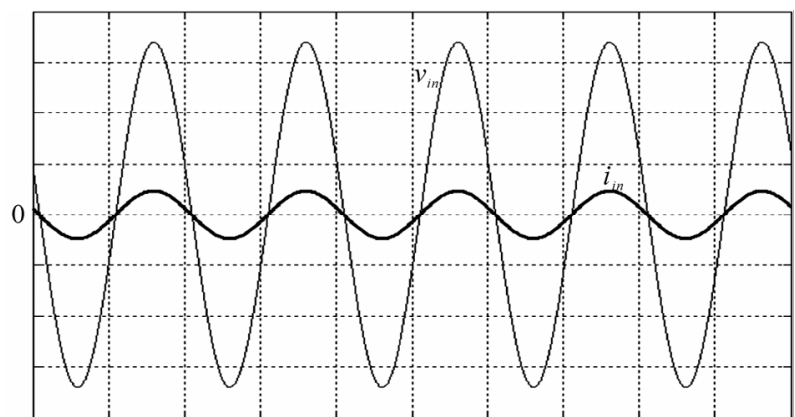

(a)

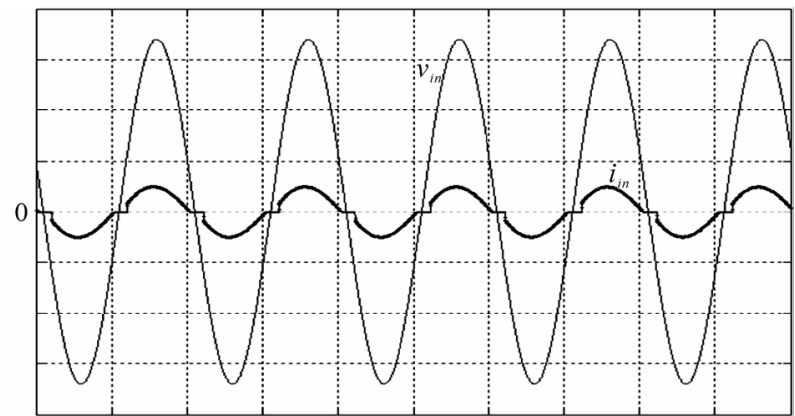

(b)

Figure 6. $v_{\text {in }}$ and $i_{\text {in }}$ waveforms for $R_{l}=900 \Omega$ (a) With repetitive controller; (b) Without repetitive controller. Horizontal axis $10 \mathrm{~ms} / \mathrm{div})$, vertical axes: voltage $(50 \mathrm{~V} / \mathrm{div})$, current $(2.5 \mathrm{~A} / \mathrm{div})$.

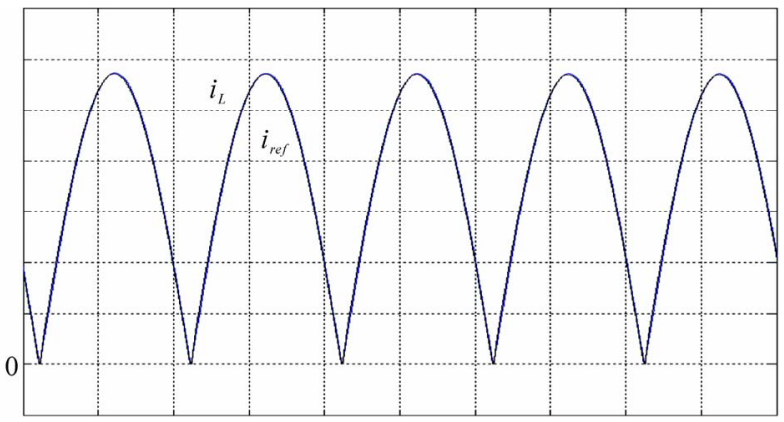

(a)

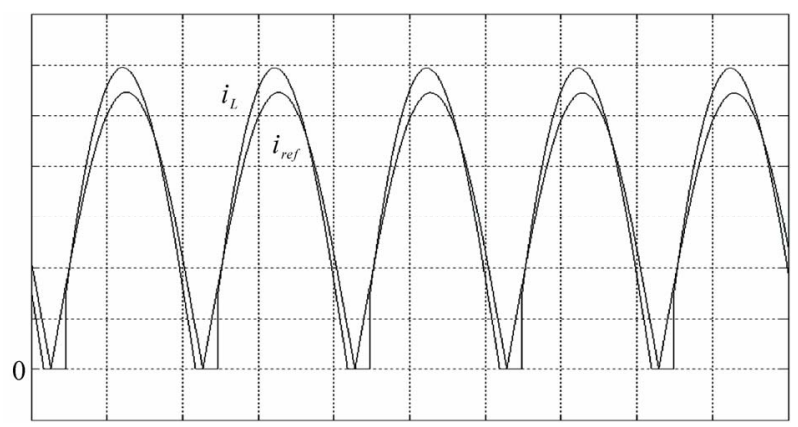

(b)

Figure 7. $i_{\text {ref }}$ and $i_{L}$ waveforms for $R_{I}=900 \Omega$. (a) With repetitive controller; (b) Without repetitive controller. Horizontal axis ( $5 \mathrm{~ms} /$ div). Vertical axis (0.2 A/div).

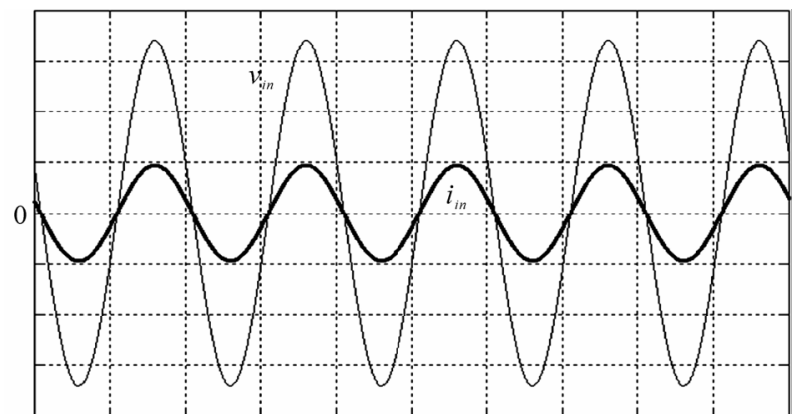

(a)

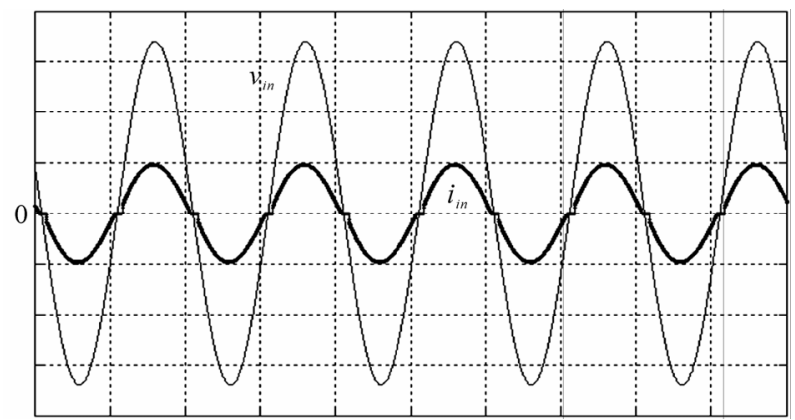

(b)

Figure 8. $v_{\text {in }}$ and $i_{\text {in }}$ waveforms for $R_{I}=450 \Omega$. (a) With repetitive controller; (b) Without repetitive controller. Horizontal axis $(10 \mathrm{~ms} / \mathrm{div})$.Vertical axes: voltage $(50 \mathrm{~V} / \mathrm{div})$, current (2.5 A/div).

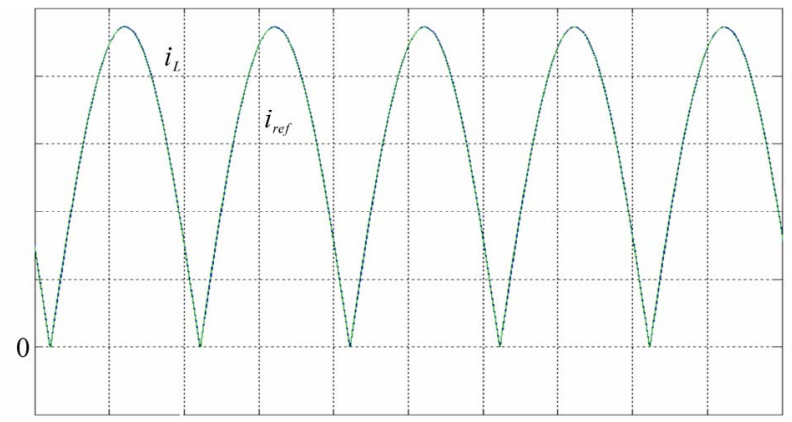

(a)

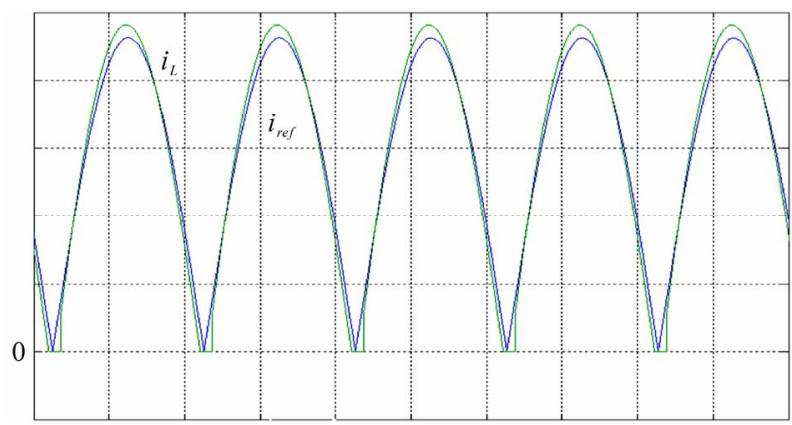

(b)

Figure 9. $i_{\text {ref }}$ and $i_{L}$ waveforms for $R_{l}=450 \Omega$. (a) With repetitive controller; (b) Without repetitive controller. Horizontal axis (5 ms/div). Vertical axis (0.5 A/div). 


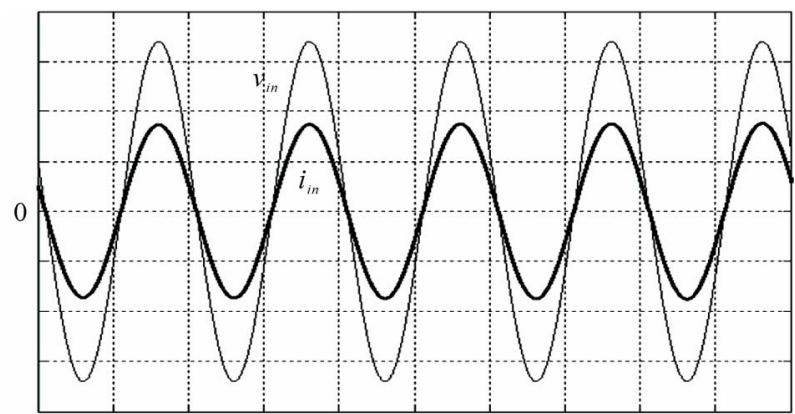

(a)

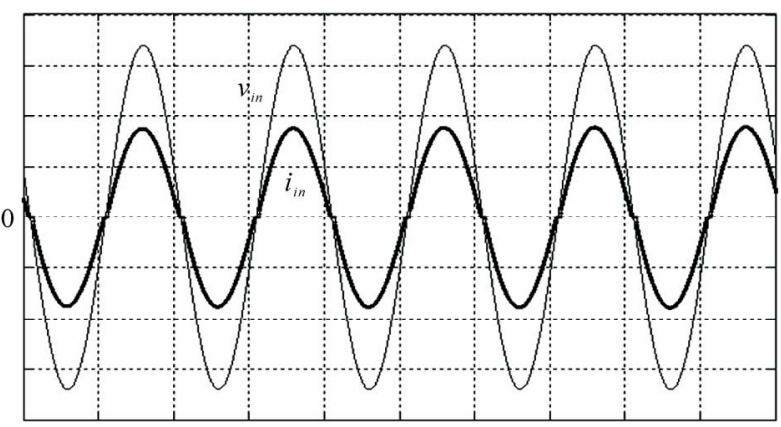

(b)

Figure 10. $v_{\text {in }}$ and $i_{\text {in }}$ waveforms for $R_{1}=225 \Omega$. (a) With repetitive controller; (b) Without repetitive controller. Horizontal axis $(10 \mathrm{~ms} /$ div $)$. Vertical axes: voltage (50 $\mathrm{V} /$ div $)$, current (2.5 A/div).

current waveforms with and without repetitive controller respectively with $R_{l}=225 \Omega$ (output power equals 400 W).

Figure 11(a) shows the reference current and inductor current waveforms for $R_{l}=225 \Omega$ when the repetitive controller is applied. It is noticed that the waveforms are coincident.

Figure 11(b) is similar to Figure 11(a) but no repetitive controller is used. No good tracking between the reference and inductor current is observed.

The power factor (PF) and the total harmonic distortion (THD) of input current with and without repetitive controller are calculated under various load and are listed in Table 2 and Table 3.

As shown in Table 2, a highly reduction in total harmonic distortion of input current is shown when a repetitive controller is used compared with Table 3 (without repetitive controller).

The waveforms of input voltage and input current for the proposed repetitive controlled PFC boost converter are plotted in Figure 12 when the load resistance $R_{l}$ is suddenly changed from $900 \Omega$ to $180 \Omega$. The input current magnitude is increased from $1.18 \mathrm{~A}$ to $5.8 \mathrm{~A}$.

We can find that the input current $i_{\text {in }}$ always in phase with the input voltage $v_{\text {in }}$ even though under transient response. Consequently, the proposed repetitive controller

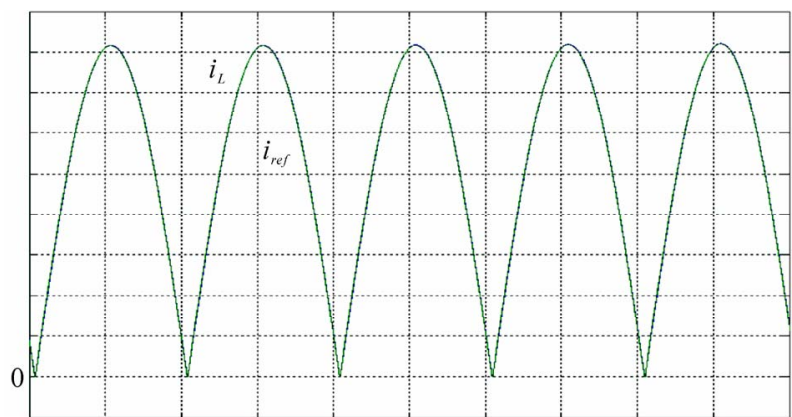

(a)

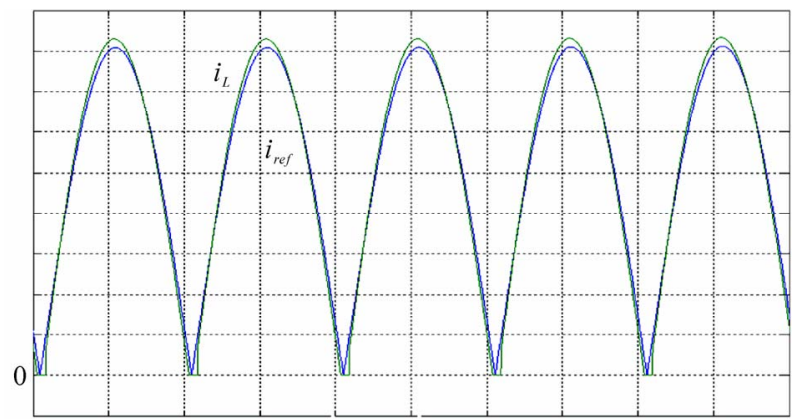

(b)

Figure 11. (a) iref and $i_{L}$ waveforms for $R_{1}=225 \Omega$ with repetitive controller. Horizontal axis $(5 \mathrm{~ms} /$ div $)$.Vertical axis (0.5A/div); (b) $i_{\text {ref }}$ and $i_{L}$ waveforms for $R_{1}=225 \Omega$ without repetitive controller. Horizontal axis $(5 \mathrm{~ms} / \mathrm{div})$. Vertical axis $(0.5 \mathrm{~A} / \mathrm{div})$.

can keep good performance under the condition of load change.

All the previous results are obtained for input voltage

Table 2. Calculated THD and PF for various loads with repetitive controller.

\begin{tabular}{cccc}
\hline$R_{l}(\Omega)$ & Output power (w) & THD (\%) & PF \\
\hline 1800 & 50 & 2.1 & 0.9992 \\
900 & 100 & 0.9 & 0.9998 \\
450 & 200 & 0.41 & 0.9999 \\
225 & 400 & 0.22 & 1 \\
\hline
\end{tabular}

Table 3. Calculated THD and PF for various loads with repetitive controller.

\begin{tabular}{cccc}
\hline$R_{l}(\Omega)$ & Output power $(\mathrm{w})$ & THD $(\%)$ & PF \\
\hline 1800 & 50 & 34.16 & 0.9965 \\
900 & 100 & 14.99 & 0.9977 \\
450 & 200 & 6.8 & 0.9992 \\
225 & 400 & 3.5 & 0.9998 \\
\hline
\end{tabular}


of $170 \mathrm{~V}$ (peak). In order to validate the repetitive controller performance for input voltage variation, the simulated steady state waveforms of inductor current $i_{L}$ and reference current $i_{\text {ref }}$ for the case of input voltage with $20 \%$ larger than the nominal value and $R_{l}=450 \Omega$ (output power equals $200 \mathrm{~W}$ ) are plotted in Figure 13. The reference current and inductor current are coincident and good tracking is also maintained and is not affected with the variation of input voltage.

Figure 14 shows the input voltage and input current

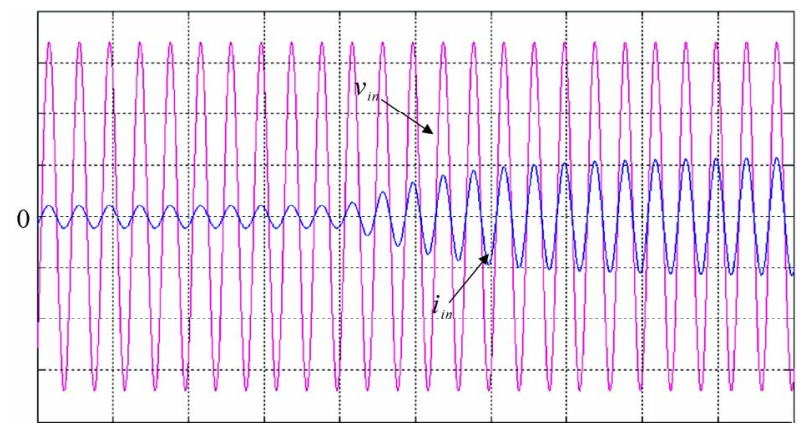

Figure 12. $v_{\text {in }}$ and $i_{\text {in }}$ waveforms with repetitive controller. $R_{l}$ is changed from $450 \Omega$ to $180 \Omega$. Horizontal axis (50 $\mathrm{ms} /$ div).Vertical axes: voltage ( $50 \mathrm{~V} / \mathrm{div})$, current ( $5 \mathrm{~A} / \mathrm{div})$.

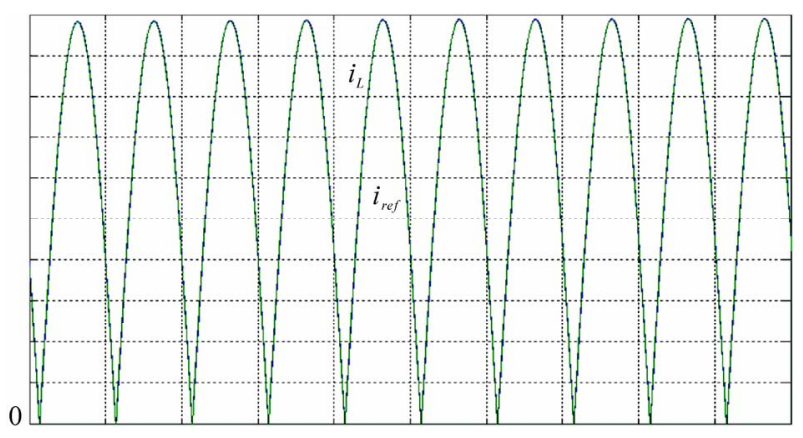

Figure 13. $i_{\text {ref }}$ and $i_{L}$ waveforms for $R_{1}=450 \Omega$ without repetitive controller. Horizontal axis $(5 \mathrm{~ms} / \mathrm{div})$. Vertical axis (0.2 A/div). Amplitude of input voltage equals $203 \mathrm{~V}$.

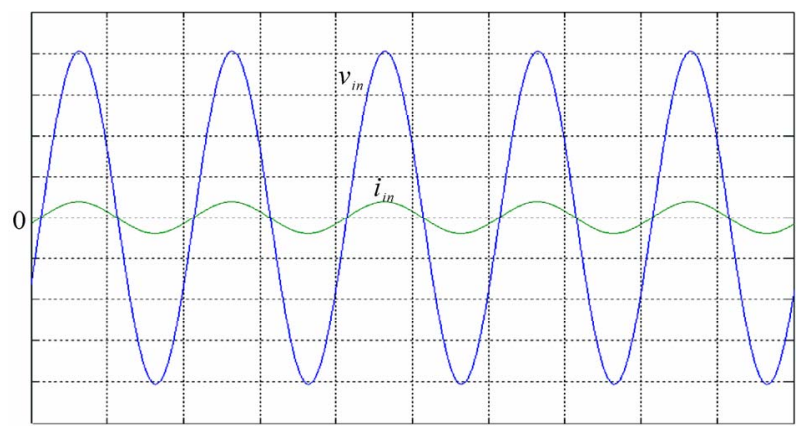

Figure 14. $v_{\text {in }}$ and $i_{\text {in }}$ waveforms for $R_{1}=450 \Omega$. with repetitive controller. Horizontal axis $(5 \mathrm{~ms} / \mathrm{div})$. Vertical axes: voltage (50 V/div), current (5 A/div). Amplitude of input voltage equals $203 \mathrm{~V}$. waveforms for the same conditions in Figure 13. No zero crossing distortion in input current is observed and the input current is in phase with the input voltage.

\section{Conclusions}

In this paper, the control approach to solve the problem of zero crossing distortion of input current of PFC boost converter has been presented. To achieve this goal, a repetitive controller is inserted in series with the PI controller of current loop. We presented a graphical design technique based on the frequency domain analysis of linear system to achieve a repetitive controller that preserves system stability. Because our approach is based on graphical inspection of the Nyquist envelop, the design procedure was simple and intuitive. A low pass filter with gain lower than one to ensure the system stability and to limit the bandwidth is included. Several simulations are performed to verify the validity of the proposed repetitive controller. The results obtained with and without repetitive controller are compared. The results with repetitive controller shows very low total harmonic distortion of input current, good tracking of reference current and inductor current (very low steady state error) and no zero crossing distortion of input current. Transit responses to step change in load are presented to exhibit the robustness of the proposed repetitive controller against load variations. The performance of the system with the proposed controller has not affected with the variation of input voltage.

\section{References}

[1] J. Sun, "On the Zero-Crossing Distortion in Single-Phase PFC Converters," IEEE Transactions on Power Electronics, Vol. 19, No. 3, 2004, pp. 685-692. doi:10.1109/TPEL.2004.826491

[2] H. C. Chen, "Duty Phase Control for single-Phase BoostType SMR," IEEE Transactions on Power Electronics, Vol. 23, No. 4, 2008, pp. 1927-1934. doi:10.1109/TPEL.2008.924627

[3] M. Chen and J. Sun, "Feedforward Current Control of Boost Single-Phase PFC Converters," IEEE Transactions on Power Electronics, Vol. 21, No. 2, 2006, pp. 338-345. doi:10.1109/TPEL.2005.869746

[4] H. C. Chen, S. H. Li and C. M. Liaw, "Switch-Mode Rectifier with Digital Robust Ripple Compensation and Current Waveform Controls," IEEE Transactions on Power Electronics, Vol. 19, No. 2, 2004, pp. 560-566. doi:10.1109/TPEL.2003.823200

[5] S. Hara, Y. Yamamoto, T. Omata and M. Nakano, "Repetitive Control System: A New Type Servo System for Periodic Exogenous Signals," IEEE Transaction on Automatic Control, Vol. 33, No. 7, 1988, pp. 659-668. doi:10.1109/9.1274 
[6] T. Inoue, "Practical Repetitive Control System Design," IEEE Proceedings of 29th Conference on Decision and control, Honolulu, 5-7 December1990, pp. 1673-1678. doi: $10.1109 /$ CDC.1990.203906

[7] B. A. France and W. M. Wonham, "The Internal Model Principle for Linear Multivariable Regulators," Applied Mathematics and Optimization, Vol. 2, No. 2, pp. 170194, 1975. doi: 10.1007/BF01447855

[8] Y. Y. Tzou, S. L. Jung, and H. C. Yeh, "Adaptive Repetitive Control of PWM Inverters for Very Low THD Ac-Voltage Regulation with Unknown Loads," IEEE Transactions on Power Electronics, Vol. 14, No. 5, 1999 , pp. 973-981. doi:10.1109/63.788503

\section{Nomenclature}

$V_{o} \quad$ Dc output voltage of boost converter.

$V_{r e f} \quad$ Reference voltage for voltage loop.

$e_{v} \quad$ Voltage error (the difference between reference and output voltage).

$G_{c v}(s) \quad$ Voltage controller transfer function.

$I_{\text {ref }} \quad$ Output of voltage controller.

$s(t) \quad$ Unity sine wave.

$i_{r e f} \quad$ Current command $\left(I_{r e f}\right.$ multiplied by $\left.s(t)\right)$.

$v_{\text {in }} \quad$ Ac input voltage to the rectifier

$\hat{v}_{i n} \quad$ Amplitude of ac input voltage.

$i_{L} \quad$ Inductor current.

$e_{i} \quad$ Current error (the difference between current command and inductor current).

$G_{c i}(s) \quad$ Current controller transfer function.
[9] G. Escobar, A. A. Valdez, J. L. Ramos and P. Mattavelli, "Repetitive-Based Controller for a UPS Inverter to Compensate Unbalance and Harmonic Distortion," IEEE Transactions on Industrial Electronics, Vol. 54, No. 1, 2007, pp. 504-510. doi:10.1109/TIE.2006.888803

[10] J. H. Moon, M. N. Lee and M. J. Chung, "Repetitive Control for the Track-Following Servo System of an Optical Disk Drive," IEEE Transactions on Control Systems Technology, Vol. 6, No. 5, 1998, pp. 663-670. doi:10.1109/87.709501

[11] K. Ogata, "Modern Control Engineering," 4th Edition, Prentice Hall, New Jersey, 2002.

$V_{\text {cont }} \quad$ Output of current controller.

$V_{t r i} \quad$ Triangular wave.

$\hat{v}_{i n} \quad$ Amplitude of triangular wave.

$i_{\text {in }} \quad$ Ac input current to the rectifier.

$L \quad$ Boosting inductance.

$d(t) \quad$ Duty ratio.

$K_{p} \quad$ Proportional gain of current controller.

$K_{i} \quad$ Integral gain of current controller.

$q(s) \quad$ Low pass filter transfer function.

$C_{R P}(s) \quad$ Transfer function of repetitive controller.

$G(s) \quad$ Open loop transfer function without repetitive controller).

$G_{R P}(\mathrm{~s}) \quad$ Open loop transfer function with repetitive controller.

$\omega_{s} \quad$ Double of input line frequency $(\mathrm{rad} / \mathrm{sec})$. 